Interfaces

«Grâce poétique » et œuvre d'art : méditations phénoménologiques dans le Journal de l'analogiste de Suzanne Lilar

\title{
Nataliya Lenina
}

\section{(2) OpenEdition}

\section{Journals}

Édition électronique

URL : http://journals.openedition.org/interfaces/670

DOI : 10.4000/interfaces.670

ISSN : 2647-6754

Éditeur :

Université de Bourgogne, Université de Paris, College of the Holy Cross

\section{Édition imprimée}

Date de publication : 12 décembre 2019

Pagination : 73-98

ISSN : 1164-6225

\section{Référence électronique}

Nataliya Lenina, « «Grâce poétique » et œuvre d'art : méditations phénoménologiques dans le Journal de l'analogiste de Suzanne Lilar », Interfaces [En ligne], 42 | 2019, mis en ligne le 12 décembre 2019, consulté le 07 janvier 2021. URL : http://journals.openedition.org/interfaces/670 ; DOI : https:// doi.org/10.4000/interfaces. 670

\section{(c) (†)}

Les contenus de la revue Interfaces sont mis à disposition selon les termes de la Licence Creative Commons Attribution 4.0 International. 


\title{
« GRÂCE POÉTIQUE » ET CEUVRE D'ART : MÉDITATIONS PHÉNOMÉNOLOGIQUES DANS LE JOURNAL DE L'ANALOGISTE DE SUZANNE LILAR
}

\author{
Nataliya Lenina \\ Université York, Collège Universitaire Glendon, Toronto
}

\begin{abstract}
Résumé
Le présent article explore le «mécanisme du fonctionnement » de la poésie libérée du verbal moyennant une analyse phénoménologique que le lecteur trouve dans le Journal de l'analogiste de Suzanne Lilar. En passant par les concepts de l'original et de la copie, l'écrivaine belge conceptualise ses propres expériences poétiques et leur " agir symbolique » instable (vécus à différentes époques de sa vie) autour des rapports dialogiques entre l'œuvre d'art, le spectateur-lecteur et l'artiste. Ce dialogue mène l'écrivaine belge à la découverte de correspondances entre les rythmes et formes naturels et les rythmes et formes artistiques.
\end{abstract}

\begin{abstract}
This article focuses on the Journal of the analogist (1954) by Suzanne Lilar, Belgian writer of Flemish origin and French expressions. Through the concepts of the original and the copy, the Belgian writer conceptualizes her own poetical experiences lived at different times in her life and their "symbolic act." The author demonstrates the importance of dialogic relationship between the artwork, the viewer-reader and artist. This dialogue leads the Belgian writer to discover the "liberated verbal" poetry of daily life, as well as the correspondence between the natural rhythms and artistic forms.
\end{abstract}

\section{Préambule}

« Le reproche le plus blessant que l'on p[uisse] faire à une œuvre, c'e[st] qu'elle ne baign[e] point dans l'état de grâce poétique » (Lilar, Journal de l'analogiste 94), note Suzanne Lilar, écrivaine belge 
d'origine flamande et d'expression française, dans son Journal de l'analogiste (paru chez Julliard en 1954). ${ }^{38}$ L'ouvrage a obtenu le prestigieux prix Sainte-Beuve la même année. Le texte du Journal révèle les grandes lignes de la pensée analogiste lilarienne qui traverse, comme un fil rouge, toute son œuvre littéraire (poèmes, romans, pièces de théâtre, écrits autobiographiques), ainsi que de nombreux essais. Quoique considéré par la critique comme son livre majeur, il n'a été réédité qu'une seule fois, chez Grasset en 1979. C'est pour cette édition que Julien Gracq écrit une préface et que Jean Tordeur rédige une introduction. Les deux écrivains soulignent à plusieurs reprises la démarche innovatrice et résolument expérimentale de cet ouvrage iconotextuel qui essaie d'élaborer des principes sous-jacents à la poésie « déconnectée du langage » (Gracq 13). La démarche de l'écrivaine belge est d'autant plus surprenante que son texte est un texte extrêmement poétique en lui-même : " on y passe à chaque instant de la poésie à la réflexion sur la poésie sans changer aucunement de palier. » (Gracq 7) Chez Lilar, la «poésie libérée » du verbal (Gracq 15), poésie de l'Être ayant partie liée avec les bases épistémologiques, met en marche simultanément le sensible et l'intelligible. Il s'agit de cette poésie du quotidien qui « se moque du goût [...] [et] des connaissances » (Lilar, Journal 94). Depuis sa tendre enfance, Lilar la retrouve dans la nature, dans le jeu, dans l'Art, dans les choses les plus ordinaires de la vie de tous les jours. Une fois touchée par cette poésie du monde, elle vit ce qu'elle-même appelle des «moments privilégiés » qui la « détach[ent] moins des choses quotidiennes que d'une façon quotidienne de les regarder » (Lilar, Une enfance gantoise 204). La poésie d'un tableau peint ou d'un morceau de musique, la poésie d'une promenade ou « la poésie de l'exemplarité » (Lilar, Journal 73) d'un chien entrevu par la fenêtre de la voiture (l'image sur laquelle s'ouvre le Journal) ne sontelles pas au fond des phénomènes du même ordre $?^{39}$ En effet, en proposant une approche poétique au monde, à l'Art, le Journal de l'analogiste nous apprend à « projet[er] sur [des] accident[s] de notre vie la lumière intemporelle de la poésie » (Lilar, Journal 154-155).

Par l'analyse d'exemples paradigmatiques présentés dans le Journal de l'analogiste de Suzanne Lilar, cet article explore des assises de la poétique de l'œuvre d'art. Comment « lire » un tableau, une sculpture, un monument architectural... ? Comment « regarder » pour voir/percevoir ce qu'ils dévoilent ? Qu'est-ce cet échange mutuel entre l'œuvre d'art et l'homme-spectateur-lecteurdécrypteur? En passant par les concepts de l'original et de la copie, l'auteur conceptualise ses propres expériences poétiques (vécus à différentes époques de sa vie) et leur " agir symbolique » instable

38 Nous emploierons plus loin dans notre texte le titre abrégé Journal.

39 « Je me souvins que les hasards de la vie quotidienne m'avaient fourni presque autant de rencontres poétiques que les arts. » (Lilar, Journal 153) 
autour des rapports dialogiques entre l'œuvre d'art, le spectateur-lecteur et l'artiste. Ce processus implique soit les « reconnaissances visuelles » (Marin 266), soit les reconnaissances conceptuelles de la part du récepteur (lecteur-spectateur) - grâce à la semiosis ad infinitum ${ }^{40}$-, soit les deux. Il mène l'écrivaine belge à la découverte des correspondances entre les rythmes et formes naturels et les rythmes et formes artistiques.

Du point de vue de la forme, le Journal de l'analogiste a une architecture complexe et résiste à toute classification. Il s'agit d'un livre illustré oscillant entre deux genres : l'écriture analytique de facture très libre (le genre d'essai à la fois littéraire, philosophique et esthétique) et l'écriture autobiographique (le titre est belle et bien évocateur !). Fondé sur les souvenirs, sur des expériences que l'auteur a vécues, l'ouvrage recèle effectivement certains traits propres à l'autobiographie. Comme dans les textes autobiographiques, la narration se fait à la première personne. Lilar, pour qui la praxis individuelle occupe une place centrale dans ses réflexions, écrit ainsi à propos de ses intentions à la fin du Journal : « J'avais pris le parti de ne pas déborder le cadre de mon expérience personnelle. » (Lilar, Journal 192) À l'instar d'une autobiographie, le texte dessine, quoique d'une manière indirecte ou, comme le dit Julien Gracq dans sa préface, " à $S A$ manière ${ }^{41}$ ", " un portrait très expressif » (préface, 12) de l'auteur. En outre, sa structure même s'approche de celle d'un journal intime. Le lecteur perçoit d'emblée que le livre est écrit de façon fragmentaire : on voit de courts passages séparés par des blancs typographiques, comme s'il s'agissait des entrées non datées d'un journal intime. D'ailleurs, on retrouve dans le livre des extraits des vrais journaux intimes de l'auteur, qui y sont cités en intratextes. ${ }^{42}$ Cependant, il ne s'agit pas d'un simple « journal » traduisant des événements au jour le jour. Il est une véritable « aventure poétique » (Lilar, Journal 112). Julien Gracq le compare plutôt à « un journal de bord », à un " journal d'une expédition de découverte » qui fraie de nouvelles voies à la perception de la poésie. Le texte progresse, comme le remarque Gracq, «par agglutinations successives [...] à la manière d'une boule de neige. » (préface, 15). Le style change au cours du Journal. L'écriture devient de plus en plus abstraite et l'ouvrage ressemble de plus en plus à un essai.

40 Par le terme de sémiosis nous entendons le processus de l'assignation de sens à un signe (entendu comme l'union du signifiant avec le signifié) ; ce processus pourrait être considéré comme ad infinitum lorsque nous prenons en considération le rôle actif du Sujet interprétant, ainsi que le contexte (culturel, social, etc.) dans lequel la sémiosis se déroule.

41 Nous soulignons.

42 Par exemple, le passage décrivant les impressions d'une promenade aux pages 141-142, tiré du journal intime de 1946, entrée datée du 22 février. 
Le Journal se termine par une composante qu'on pourrait considérer comme une annexe s'il n'y avait pas à sa fin l'inscription « fin du journal ». Là, on retrouve des souvenirs dits « condensés » (toujours bien précis), ainsi que beaucoup d'intertextes. Cette partie est présentée sous le titre « Récréations analogiques » qui la sépare clairement du texte précédent. La partie finale se distingue aussi typographiquement du reste du livre : elle est mise en page en caractères plus petits que ceux employés pour la partie dite principale. Par ailleurs, ces passages sont découpés non seulement par le blanc du papier, mais aussi par des culs-de-lampe, vignettes qui marquent, habituellement, les hiatus. Ainsi, ces petits textes sont introduits l'un par rapport à l'autre sous le signe de la rupture, c'est-à-dire qu'ils se présentent, avant tout, en tant que textes autonomes, mais liés par des thèmes communs.

L'image se présente dans le livre sous différentes formes. Elle apparaît de la manière la plus directe quand on la voit sous sa forme iconique : plusieurs illustrations photographiques, représentant des détails des œuvres d'art, sont insérées entre les pages du texte, au milieu du livre. L'image est aussi explicitement présente dans le texte par le biais du verbal (par l'ekphrasis notamment).

\section{La poésie étrange des « formes rebutantes "}

\section{Ornements muraux de la crypte des Capucins}

La première illustration photographique du Journal représente un détail de la décoration murale faite d'ossements humains de la crypte des Capucins. S'il n'y avait pas de description du lieu aux pages 89-90 du Journal et d'inscription dans la « Table des planches », le lecteur aurait des difficultés à reconnaître ce qui figure dans cette photo, ainsi que la matière dont les ornements sont fabriqués. On ne sait pas au juste quel type de lecture de l'image il faut choisir : une lecture horizontale ou verticale. En l'occurrence, si le lecteur tourne la photographie dans la mauvaise direction, il risque d'y voir, par exemple, des grains de verre arrangés selon un motif floral sur une étoffe (fig. 1). En fait, la photo montre une véritable transformation du squelette humain en « fleurons et en acanthes pour aborder le monde abstrait de l'ornemental » (Journal 90). L'image iconique de la crypte des Capucins et sa description peuvent nous faire penser à l'esthétique du carnavalesque chez Bakhtine, où l'ordre établi est transgressé et les valeurs conventionnelles sont renversées. Ces images renvoient en effet moins au pathétique de la mort qu'à son effacement devant le carnavalesque. Bien évidemment, le spectacle, comme le note Lilar, " relève assez bassement du macabre » (Lilar, Journal 89), mais en même temps, il engendre une «étrange poésie » (Lilar, Journal 90) qui « libèr[e] momentanément de l'humain et jett[e] au-delà, dans la confusion de la création » (90). Le décor d'ossements rend visible 


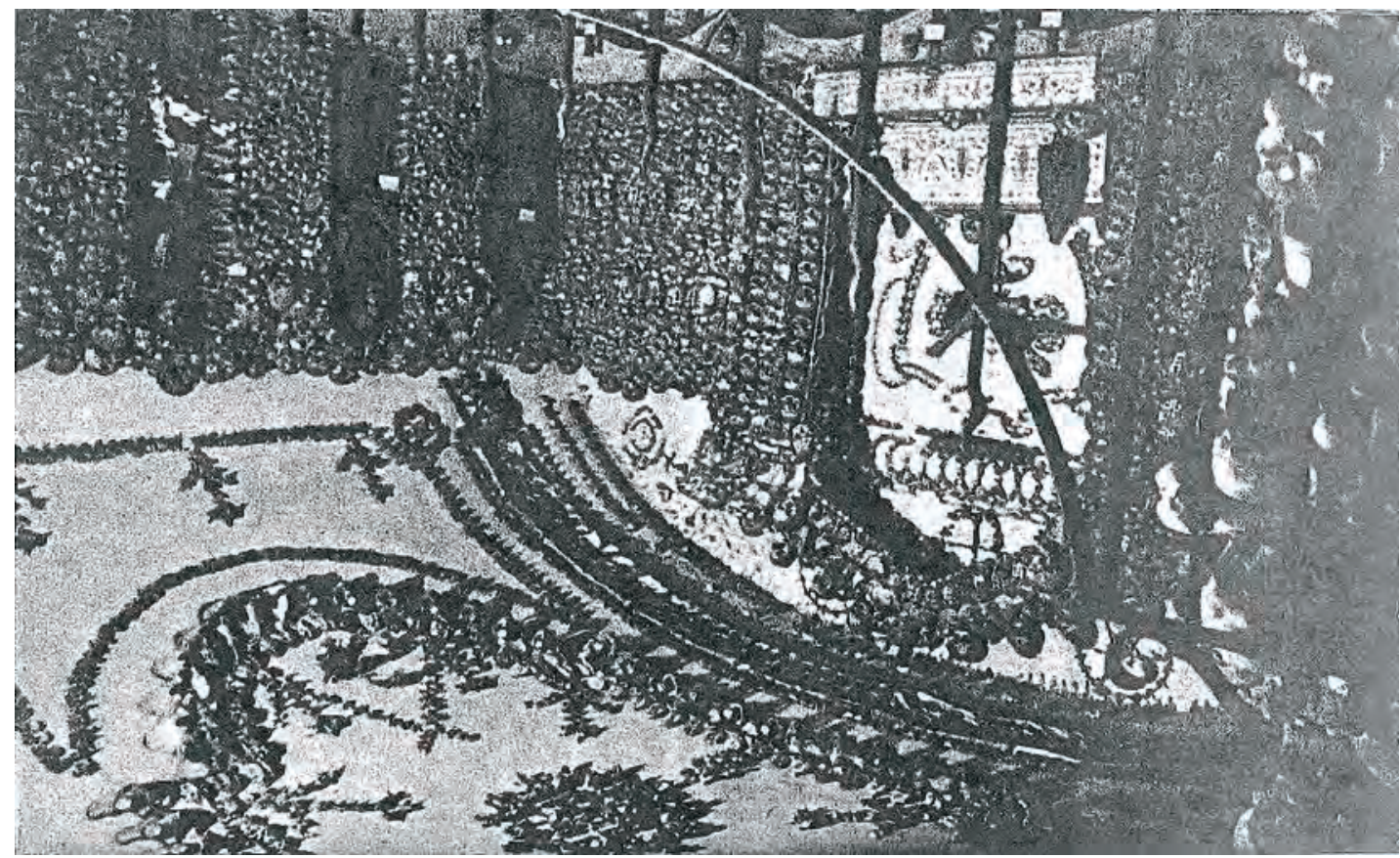

Fig. 1. Ornements muraux de la crypte des Capucins. Cette photographie figure seulement dans l'édition du Journal de l'analogiste chez Julliard (1979).

le processus de la métamorphose pendant lequel une forme se montre susceptible de reparaître sous une nouvelle forme tout en restant essentiellement la même. S'agit-il d'une sorte de réconciliation de la vie avec la mort? La démarche de l'écrivaine belge ne vise-t-elle pas au bout du compte une réconciliation avec la mort redoutée. Au fil des pages de l'autobiographie de Lilar Une enfance gantoise, on apprend que " la mort qui est épouvante » peut être aussi " fascination et suavité » (Lilar, Une Enfance gantoise 122). Ces sentiments contradictoires à l'égard de la mort, Lilar les a hérités de sa mère, qui, fascinée par la mort, en «bonne élève » de Montaigne, initie sa fille à la réalité de la mort, sans l'édulcorer :

Très tôt Maman avait voulu me familiariser avec le spectacle de la mort et choisissant pour le faire une occasion bénigne, elle m'engagea à aller voir la chatte Mina, morte de vieillesse 
chez ma grand-mère à l'âge de dix-huit ans. Je me revois descendant l'escalier de la cave. Je m'efforçais au courage. Je prenais sur moi. Mina était étendue dans son panier comme endormie. Mais quelque chose dissuadait d'y croire et dénonçait, sous l'apparence du sommeil, une opération sournoise. Bonne-Maman m'y invitant, je caressai la chatte qui avait maintenant la consistance du bois. Mais je ne me laissai pas retenir par l'étrangeté de cette découverte, l'attention captée par quelque chose que je percevais comme une présence. C'était la présence investissante quoique diffuse de la mort. (Lilar, Une Enfance gantoise 124)

L'absence était perçue comme une présence, la mort comme un sommeil. Ce " spectacle », grâce auquel l'enfant découvre de fausses apparences, la bouleverse et incite à approfondir ses réflexions. Le mot « spectacle » qu'emploie Lilar pour désigner les manifestations de la mort est encore plus applicable pour le passage qui parle du « pathétique bouffon » de l'agonie imaginaire de son oncle. Des images atroces de la mort hypothétique de son oncle (qui, étant gravement malade, séjournait chez la famille Verbist) terrorisaient la petite fille :

J'imaginais le pathétique bouffon de ce corps se défendant contre la mort, les sursauts de cette énorme chair rebelle à se laisser prendre, j'imaginais l'agonie, le passage de vie à trépas, le mourir pour parler comme Montaigne. Je n'ai pas cessé de le trouver effroyable et de le distinguer de la grande paix fraternelle de la mort. Au chevet de mes morts les plus chéris, il $\mathrm{y}$ eut toujours ce moment atroce où je les abandonnai, où je les laissai se débattre seuls, où m'affaissant, je me suis remise sur un tiers, religieuse ou infirmière, de ces soins qu'un instant auparavant je prodiguais avec tant de ferveur. Toujours il y eut cette désertion, cette minute où je les ai trahis et tandis qu'eux se battaient encore contre la mort, moi je l'espérais, je pariais pour elle. (Lilar, Une enfance gantoise 128)

La petite fille commence à « aimer » la mort, ${ }^{43}$ et même, entre dans une sorte de connivence avec elle regardant toujours au-delà de la mort. Intelligemment, elle la pratique, comme le conseille le sage Montaigne. Parfois, le lecteur pourrait être étonné par la lucidité du regard de la fillette devant la mort ou choqué par son sang-froid. Elle aide, par exemple, à « achever» les oiseaux blessés par le chat dans le jardin public pour les délivrer de la souffrance ; elle aide également son chien malade à mourir en diminuant la durée de l'agonie. Cette « connivence » dont parle Lilar n'interdit en aucun cas l'effroi devant « le guignol de la mort », devant « les grimaces et convulsions qui l'accompagnent» (Lilar, Une enfance gantoise 125), mais, sans fausse et humiliante pitié, enseigne à réfléchir sur la « dialectique »

43 « J'aimais la mort. J'aimais rêver de mes morts. » (Lilar, Une enfance gantoise 125). 
de la mort justement pour vivre bien, joyeusement et dignement, donnant à la mort son droit. Et si l'œuvre de Lilar est traversée par la mort, c'est pour témoigner de l'amour insatiable de la vie.

\section{La Villa d'Este}

Dans le cadre du Journal, l'image de la crypte des Capucins renvoie aussi à la représentation poétisée de la Villa d'Este et de ses magnifiques jardins (chef-d'œuvre de l'architecture italienne du XVI'e siècle, à Tivoli, près de Rome). L'image de ce lieu apparaît dans le texte sous la forme d'une longue description enchâssée dans le souvenir de la visite du site par l'auteur (Lilar, Journal 86-88), lui-même précédé d'un bref souvenir d'enfance décrivant des « sensations étranges » et du plaisir que goûtait la petite Suzanne à contempler « la trace d'une ammonite ou d'une fougère incrustée dans la pierre » (Lilar, Journal 85). Ainsi, à travers une allusion à la notion de métamorphose, ce souvenir annonce la description de la Villa, l'exemple enchanteur d' " une métamorphose sans truquage » (Lilar, Journal 86). Surtout, ce phénomène était très visible dans les jardins où « la nature saisit, pétrit, refaçonne » (Lilar, Journal 88) les formes en marbre, où tout se défait et se désagrège « sous l'assaut » $(88)$ du monde végétal. Le travail de l'architecte et du sculpteur a été repris par la nature et, « [d]ans ce foisonnement de contradictions où l'effusion funèbre s'accordait au rythme de la vie, tout était remis en question » (Lilar, Journal 101). C'est comme si la nature voulait retrouver la forme initiale de la matière. Est-ce de ce combat de deux forces antagonistes que naît cette « étrange poésie » (semblable à celle de la crypte des Capucins) qui apporte un sentiment de sérénité en libérant l'espace (par le mouvement créateur de la transformation) d'un état morbide?

La Diane d'Ephèse s'efface et semble rentrer dans la pierre. Le corps d'un jeune Bacchus, rongé comme celui d'un ascète, est couvert des pustules grisâtres du lichen. Un Neptune, voilé jusqu'au menton d'un fin réseau de saxifrage, a pris avec sa toison de verdure, l'aspect débonnaire d'un caniche. Le tridacne de la fontaine du Bicchierone, étroitement enserré par une mousse géante, lui dispute désespérément chaque saillie, chaque rainure, chaque filet de son extravagante coquille. Où finit le marbre, où commence le sournois cancer végétal, on ne sait plus. Où l'invention du Bernin, où les retouches insidieuses de la nature ? Une statue intacte, préservée, peut-être belle, laisse curieusement indifférent. Pourquoi ? Serait-ce que la beauté fascine moins que la désagrégation de la beauté ? Et nous deviendrait-elle précieuse dans la mesure où nous la savons périssable? (Lilar, Journal 88)

Ces questions, colorées par le goût pour le difforme et le grotesque, parcourent toute l'œuvre littéraire de Lilar : " J'étais trop flamande, écrit-elle, pour ne pas subir la fascination d'un art adonné à la 
monstruosité, à l'anomalie, à la distorsion des formes » (Journal 100) ; « [M]on côté flamand : mon besoin de laideur, d'anormalité, de monstruosité : mon impression alors de remise en place. J'échappais au monde du joli. » (Inédits, Écrits à caractère autobiographiques).$^{44}$ Les chapeaux de folie, les masques de carnaval, plus encore les infirmes et les fous, les monstres de Bosch, de Grünewald ou de Goya l'attirent mystiquement. En les contemplant, l'écrivaine belge y rejoint sa propre « logique de rêve », ce « carnavalesque » bakhtinien qui dépasse la logique du « comme il faut» et, par conséquent, « ne se réalise pleinement qu'en marge de la culture officielle » (Kristeva 83) :

[J]'aimais le spectacle des anormaux de Lourdes, ceux de Geel où les fous vivent chez l'habitant, j'aimais leur démarche saccadée et parcourue de tics, eux aussi involontaires. Avec les autres enfants je poursuivais Zot Sofietje, non pour m'en moquer comme mes compagnes mais pour jouir de son étrange comportement. J'ai compris plus tard la nature du plaisir que j'éprouvais. Assistant à une fête de gymnastique où, par charité, un anormal avait été admis dans le groupe des athlètes, je ressentis, après un bref malaise, l'étonnement d'une délivrance. Ce dément paraissait habité par quelque chose qui le dépassait. Il y avait dans le déclenchement brusque de ses gestes, dans l'égarement de son regard une sauvagerie, un emportement, un délire qui communiquaient une ivresse, faisant apparaître chez les autres, les normaux, le manque d'envol, l'empêtrement du terrestre dans ce qu'il peut avoir d'étriqué, de réduit au prévisible. (Lilar, Une enfance gantoise 127-128)

Ainsi, des spectacles avec des « anormaux » montrent le manque chez les « normaux », ce « manque d'envol » qui empêtre dans la vie terre à terre. C'est pourquoi, lors de pareils spectacles, la petite Suzanne apprend à jouir de ce qui ne se trouve pas réduit au prévisible; elle apprend à voir le monde non pas seulement à l'échelle de ses habitudes. C'est presque de la même manière que l'écrivaine jouit de la poésie d'une métamorphose imprévisible du parc de la Villa d'Este. La description de ce lieu magique hors du temps se termine par un passage où chaque phrase pèse lourd :

Il y a dans toute métamorphose, mort, naissance, quelque chose d'insaisissable. La science explique, la philosophie commente. On reste au pied de l'essentiel. Mais la poésie fait faire le saut. Elle éclaire nos curiosités et nous découvre les raisons du saisissement que nous éprouvons à surprendre - si parfaitement encadrée par la pompe baroque de la Villa d'Este la mort étroitement accolée à la vie, à les concevoir tout à coup comme de simples apparences d'un même devenir. (Lilar, Journal 88-89) (Nous soulignons.)

44 Inédits de Suzanne Lilar : Écrits à caractère autobiographiques : Fragments divers : Lilar sur elle-même et sur son œuvre (Archives, ML 08492/0024). 


\section{Les Vieilles de Goya. L'espace pictural}

La représentation des Vieilles de Goya (Lilar, Journal 159) s'inscrit bien dans la lignée des images troublantes provoquées par la crypte des Capucins. Les Vieilles épousent d'une manière ostentatoire la pensée de Lilar que la mort et la vie, " étroitement accolée ", peuvent être perçues (sans être confondues) «comme de simples apparences d'un même devenir » (Lilar, Journal 89). En même temps, ce tableau, semble-t-il, reflète " visuellement » l'esthétique du carnavalesque bakhtinien. Le tableau renvoie, d'ailleurs, au symbolisme des figurines en terre cuite des vieilles femmes enceintes et riantes se trouvant au Musée de l'Ermitage dont Mikhaïl Bakhtine parle dans L'Euvre de François Rabelais. Bakhtine souligne l'ambivalence et l'expressivité de ce grotesque mis en forme :

[...] [C]'est la mort enceinte, la mort qui donne le jour. Il n'y a rien de parachevé, de stable et de paisible dans le corps de ces vieilles femmes. [...] La vie est révélée dans son processus ambivalent, intérieurement contradictoire. Rien n'est tout prêt, c'est l'inachèvement même. (Bakhtine 35)

Ces propos sont en effet applicables à la poétique complexe et contradictoire des vieilles femmes chez Goya : par la destruction du stable, par la provocation et la négation des apparences, elle dirige l'esprit vers la « re-naissance », vers le renouveau, vers l'« éternel devenir».

La toile de Goya montre la vieillesse caricaturée, où « le paraître est plaqué sur l'être » (Louvel 153) : sous l'apparence des artifices (des bijoux, des habits, du maquillage) se cachent les corps affaissés, presque squelettiques, des deux vieilles femmes. Deux « monstres dérisoirement parés » (Lilar, Journal 159). Le message que l'image fait passer n'est-il pas l'écho de ce memento mori des vanités du XVII ${ }^{\mathrm{e}}$ siècle qui nous rappellent que personne n'échappe à la mort et que l'on ne sait jamais l'heure de ce passage ? L'image d'un homme avec un balai à l'arrière-plan incarne apparemment la mort elle-même. Ces deux personnages paraissent ridicules au spectateur « omniscient » qui voit derrière leurs épaules cette redoutable figure, capable de " balayer » (ou peut-être déjà en train de «balayer ») ces deux coquettes du monde terrestre. Or, la description dans le Journal ignore sa présence dans le tableau et se concentre principalement sur les deux figures féminines.

La « translation picturale » (Louvel 88) du tableau dans le Journal (c'est-à-dire la traduction d'un code sémiologique à un autre) est construite par antithèse : tous les éléments sont vivement contrastés soit sur le mode de la rupture soit sur le mode du prolongement. Par exemple, les «paupières sanguinolentes » de l'une des femmes s'opposent aux « orbites charbonneuses » de l'autre, « une 
boîte crânienne écrasée, épatée, ouverte sur l'orifice béant des narines » (Lilar, Journal 159) de l'une, contraste avec le crâne « grotesquement busqu[é], recourb[é] sur le clapet du bec » de l'autre (159). Mais ces contrastes complètent la même image de la sénilité de la vieillesse. Alors que ces mêmes détails de la description contrastent violemment (et cette fois-ci sur le mode de rupture) avec la beauté des robes (la blancheur de la gaze et le « bleu[] si doux [...] [des] « vaporeuses dentelles » [Lilar, Journal 160]) et l'exubérance des accessoires (pierreries, rubans, bandelettes). Pourtant, les oppositions entre les couleurs et les valeurs, entre la décrépitude des corps et la fraîcheur et l'extravagance des parures, non seulement dénoncent le jeu des apparences trompeuses, mais vont au-delà de cette fonction. La peinture dépasse de beaucoup toutes ces visées, ou «messages », relevant d'une analyse rationnelle que nous venons de signaler ci-dessus : elle entraîne le lecteur-spectateur dans une entreprise proprement poétique, elle le mène " en un lieu où les contraires cessent d'être perçus contradictoirement » (Lilar, Journal 137).

Lilar souligne dans le Journal que nulle œuvre ne lui avait révélé autant de secrets de la " poésie de l'atroce » (Lilar, Journal 159) comme ce tableau de Goya, perçu, avant tout, comme une « provocation personnelle » (159). Toutefois, le chemin pour saisir sa poésie a été fort long. Avant que la vraie rencontre avec le chef-d'œuvre n'ait lieu, Lilar s'en détournait « comme d'une inconvenance » (159). Et pourtant, elle n'a pas pu se débarrasser des sentiments contradictoires qui l'envahissaient et qui « voulai[ent] être résolu[s] » (Lilar, Journal 160). La toile tout ensemble la « happait » (159) et provoquait l'horreur; elle se sentait regardée par le tableau. Il s'agit de ce triple échange entre l'artiste, le sujet regardant et le tableau dont parle Jeanette Winterson dans son essai portant sur les objets artistiques et Merleau-Ponty dans « Le langage indirect et voix du silence » :

I move gingerly around the paintings I own because I know they are looking at me as closely as I am looking at them. There is a constant exchange of emotion between us, between the three of us ; the artist [...], the painting in its own right, and me, the one who loves it and can no longer live independent of it. The triangle of exchange alters, is fluid, is subtle, is profound and is one of those unverifiable facts that anyone who cares for painting soon discovers. [...] I think of something I did, the picture catches me, adds to the thought, changes the meaning of thought and past. The totality of the picture comments on the totality of what I am. The greater the picture the more complete this process is. Process, the energy in being, the refusal of finality, which is not the same thing as the refusal of completeness, sets art, all art, apart from the end-stop world that is always calling 'Time Please!'. (Winterson 19) 
L'œuvre accomplie n'est donc pas celle qui existe en soi comme une chose, mais celle qui atteint son spectateur, l'invite à reprendre le geste qui l'a créée et, sautant les intermédiaires, sans autre guide qu'un mouvement de la ligne inventée, un tracé presque incorporel, à rejoindre le monde silencieux du peintre, désormais proféré et accessible. (Merleau-Ponty, « Langage indirect et voix du silence »1479)

Le texte du Journal explique également que la rencontre dépend essentiellement du regard du spectateur. Comme le note Éliane Escoubas, dans un ouvrage consacré à l'espace pictural, « ce que peint la peinture, ce que donne à "voir" l'espace du tableau, c'est la mise-en-œuvre du regard, la mise en œuvre de l'exercice-extase du regard $»^{45}$ :

La peinture fait voir ce qu'on ne voit pas ordinairement - ce qu'on ne voit pas $d u$ tout : elle peint, à chaque fois, la naissance du monde sous le regard — ce qui a toujours déjà commencé lorsqu'on commence à voir ce qu'il y a à voir. (Escoubas 25 )

Escoubas se réfère ici à un passage très connu de Merleau-Ponty cité généralement dans les essais portant sur l'image, à savoir :

Je serais bien en peine de dire où est le tableau que je regarde. Car je ne le regarde pas comme on regarde une chose, je ne le fixe pas en son lieu, mon regard erre en lui comme dans les nimbes de l'être, je vois selon ou avec lui plutôt que je ne le vois. (Merleau-Ponty, L'Eil et l'Esprit 1596) (Nous soulignons.)

Comme le note Escoubas, les expressions « voir selon ou voir avec » « marquent l'écart entre la chose en tant que chose et la chose en tant que tableau » (Escoubas 15). Autrement, le tableau ne dévoile rien, ne révèle pas, mais se présente simplement en tant qu'espace de la représentation : « Une forme n'est rien en soi. Une forme n'est pas, elle existe. » (Maldiney, «L'esthétique des rythmes » 163), écrit le philosophe-phénoménologue français Henri Maldiney.

En parcourant les pages du Journal de l'analogiste, on voit l'auteur belge pratiquer régulièrement cet « exercice-extase du regard » « selon » et « avec» le tableau peint, comme le propose

45 On cite la note d'Éliane Escoubas présentée en bas de la page 16 de L'Espace pictural : « On prendra ces termes au sens étymologique : Exercice vient du latin ex-arceo, où arceo dit : contenir dans des limites, enfermer, arrêter, empêcher d'avancer (des troupes) ; ex-arceo dit alors : mettre ou tenir des troupes en mouvement, ne pas rester en repos, faire sortir des limites où (les troupes) étaient arrêtées. Extase vient du grec ek-stasis : être hors de soi, faire sortir de soi, faire changer de place. » 
Merleau-Ponty. Dans le cas des Vieilles, le texte dit qu'il faut « suivre le poète à travers la séduction des formes [...] dans la difformité, dans la laideur » (Lilar, Journal 157) et le suivre en poète. Le malaise que l'image de Goya provoque " réclame impérieusement le soulagement poétique » (Lilar, Journal 151). La transfiguration des formes se réalise sous le regard " poétique » du spectateur (le sujet regardant) qui les dote d'un «nouveau pouvoir imageant » (Lilar, Journal 164). En l'occurrence, et puisqu'il s'agit de peinture, on doit regarder ce tableau « selon la couleur » (Lilar, Journal 160) et selon les effets des contrastes, allant au-delà de la forme. Auquel cas, le spectateur se déplace sur « un plan où les extravagances de la décrépitude peuvent être inhumainement, sereinement regardées » (160). C'est le miroitement des contrastes des formes et des valeurs mis en parallèle avec le jeu des couleurs qui fait rompre « l'automatisme de la représentation » (Lilar, Journal 164) :

À ce prix, cette vieillesse redoutée [...] se dépouill[e] de ses cruautés particulières pour rentrer dans le destin universel des métamorphoses. Et ce n'est pas [...] sans intuition de ces transmutations que Goya [mêla] éclat et disgrâce, fraîcheur et décomposition [...] ; et même, allant plus loin encore dans la négation de l'apparence, qu'il avait dans le rouge d'un enrubannement de satin établi un écho au rouge répugnant des paupières sanieuses ; mais précisément ce rouge n'était pas répugnant, le peintre nous le prouvait en l'utilisant pour parure. Ainsi l'une après l'autre désarmait-il nos préventions et il n'était aucun détail si affreux fût-il — de cette peinture admirable qui ne vînt en fin de compte s'apaiser et se résorber dans l'enchantement de la couleur. (Lilar, Journal 160) (Nous soulignons.)

Cet enchantement, cet équilibre sont retrouvés à la fois grâce à une « tension particulière » (Lilar, Journal 158) créée par les «formes rebutantes » (158) qui nous projettent avec violence au-delà d'elles-mêmes et grâce au regard «poétique » du spectateur. C'est exactement le même processus que nous avons pu observer dans le cas des décorations en ossements humains de la crypte des Capucins. Lilar note que de telles œuvres d'art détiennent « le singulier pouvoir » sur le spectateur, et même, sur un point, elle leur accorde un avantage, " celui de ne pas créer de malentendu » (Lilar, Journal 158). La « franchise » de leur provocation nous garde de la passivité, d'une sorte d' " hébétude amoureuse » (158) qui aurait pu être ressentie facilement devant les formes dites trop belles, "formes suaves » (158), comme les nomme le texte du Journal.

Ainsi, chez les Vieilles de Goya, la poésie manifeste le " pouvoir de délivrance » (Lilar, Journal 162) qui se trouve renforcé " en proportion de la distance qui sépare le terrible de l'acquiescement au terrible » (162) : « [c]'est dans l'écart [...] entre la sensation et son indice poétique que résidait l'efficacité des formes agressives » (162). L'écart que les «formes rebutantes » donnent 
à franchir confère à la poésie, qui naît du dialogue entre l'œuvre (le regardé) et le spectateur (le sujet regardant), " un relief particulier » (Lilar, Journal 164). Tout compte fait, deux extrêmes, le beau et le monstrueux, se trouvent dépassés ; le macabre devient le symétriquement inverse. Le spectateur est ainsi " entraîné » (Lilar, Journal 163) par la surprise de cette "poésie de sursaut » (163) et non plus rebuté ou choqué par les formes figurées sur le tableau. La mise en œuvre de «l'exercice-extase du regard » subvertit la stabilité apparente de la réalité invitant à « une relecture phénoménologique de la catégorie de la réalité » (Escoubas 30). Cette subversion déplace ainsi la notion de la réalité « de l'ordre de l'objectivation à celui de la communication » (30). Comme le constate Maldiney, « Est réel ce qui se donne dans la rencontre. » (Maldiney 173)

\section{Rythmes internes des œuvres d'art : du particulier à l'universel à travers l'expérience du poète}

Fascinée par le trompe-l'œil, Suzanne Lilar y consacre maintes pages dans le Journal de l'analogiste. Tout en suggérant « la troublante imprécision de la poésie » (Lilar, Journal 106), ce genre pictural lui apporte les découvertes analogues les plus nombreuses :

[L]e trompe-l'œil, ne nous propose pas un simple plaisir visuel copié sur ceux que nous offre la nature ni même un plaisir de virtuosité, mais un plaisir métaphysique, celui de faire entrer les objets de notre contemplation dans un système d'analogies. Je devais voir plus tard, écrit l'auteur belge, que c'est le propre de toute entreprise poétique. [...] À vrai dire, des années s'écouleraient avant que je fusse en mesure de discerner et d'identifier les rythmes, car c'est d'eux qu'il s'agissait et, de leur appliquer la réflexion analogique. (Lilar, Journal 108-109)

Lilar termine ce passage par l'introduction de la notion de rythme, applicable non seulement au domaine musical, mais aussi à la peinture, à la sculpture, etc. Elle contemple des tableaux anciens des grands Flamands et se rend compte que la couleur, pareillement à un son, "s'évalue dans une suite " (Lilar, Journal 172-173). Cependant, la couleur, contrairement au son qui, lui, se déploie dans le temps, se trouve "découpée" (Lilar, Journal 173) dans l'espace. La couleur apparaît dans le tableau « à la fois en étendue et en profondeur, [...] [et] comme le lieu de croisement d'une série de rythmes » (173). L'espace pictural est ainsi rythmé, comme un morceau de musique ou un poème et, ses rythmes entrent en relation directe avec le spectateur :

Il y avait des rythmes plastiques en dehors des arts du même nom, et inversement des rythmes d'intensité en dehors de la musique — et la chose va de soi pour la danse et la poésie [...]. 
Je savais, écrit Lilar, que la première prise de possession du poète [...] se traduisait par un rythme figuratif de l'émotion, schéma de l'onde vivante convertie en motif et qui, fidèle représentation des mouvements du cœur dont il était issu, allait projeter à travers le poème ou la musique l'afflux nourricier d'une circulation poétique, perceptible à la fois comme un battement et comme une suite de révolutions cycliques susceptibles [...]. (Lilar, Journal 192193 ; nous soulignons.)

Ainsi, le texte signale que le propre de tout œuvre d'art est d'être traversée par des rythmes nonarbitraires, bien structurés et fondés sur l'analogie. Ses propos s'entrecroisent avec la définition du rythme artistique que propose Henri Maldiney : « le rythme est l'essence de l'art et il est son existence, étant l'acte du style » (Maldiney, «L'esthétique des rythmes » 172). L'acte du style, car issu du cœur de l'artiste, il représente ou traduit son âme, ses émotions, interprète des mouvements de son cœur.

Nous proposons d'étudier quelques expériences poétiques de l'auteur qui mettent en évidence le fait que les œuvres d'art possèdent des rythmes intérieurs, uniques et non fortuits ; ces rythmes « s'étend[ent] bien au-delà du visible » (Lilar, Journal 178) et rejoignent des rythmes et formes naturels.

\section{Les images radiographiques du portrait de Hendrickje Stoffels (Rembrandt) et du Polyptyque de Van Eyck}

Le premier exemple concerne le Portrait de Hendrickje Stoffels du pinceau de Rembrandt et montre que l'œuvre d'art se présente comme un entrelacement du visible avec l'invisible, que l'authenticité n'est pas reproductible et que le rythme interne de l'œuvre d'art peut nous aider à distinguer une copie de l'original. Lilar a profité d'une occasion d'examiner et de comparer une image radiographique de l'original d'Hendrickje Stoffels avec « celle d'une excellente copie d'époque probablement exécutée dans l'atelier de l'artiste » (Lilar, Journal 177). Cette copie a été peinte d'une manière parfaite, et le copiste aurait pu être Rembrandt, grâce à la maîtrise de son art égale à celle du grand peintre hollandais. Mais la copie n'a pas supporté l'épreuve des rayons X. En effet, la radiographie trahissait le faux. Il y avait dans ce tableau si bien imité quelque chose qui empêchait de reconnaître le pinceau de Rembrandt :46

46 La pensée esthétique de Lilar rejoint ici celle de Walter Benjamin, pour qui « la véritable œuvre d'art se laisse reconnaître à son "aura", ce rayon de présence qui émane de l'œuvre et qui est la marque qu'elle est bien venue au visible et au dicible d'un être suprasensible [...]. » (Wunenburger 178). D'après Benjamin, "À la plus parfaite reproduction il manquera toujours une chose : le hic et nunc de l'œuvre d'art - l'unicité de son existence au lieu où elle se trouve". (Benjamin 273) "Le hic et nunc de l'original constitue ce qu'on appelle son authenticité. [...] Tout ce qui relève de l'authenticité échappe à la reproduction - et bien entendu pas seulement à la reproduction technique. » (Benjamin 274) 
[L]a copie se muait en une sorte de masque carnavalesque, dépourvu de toute spiritualité et même d'expressions [...]. C'est qu'il avait été peint de l'extérieur, et si parfaite, si mystifiante que fût en lumière directe sa ressemblance, au point que l'attribution à Rembrandt avait longtemps été tenue pour probable, cette hypothèse s'écroulait sous le pouvoir pénétrant des rayons, car la ressemblance n'avait pas été travaillée en profondeur. En effet, le copiste, s'en tenant à l'imitation consciencieuse mais superficielle de l'œuvre, s'était montré impuissant à reproduire son rythme interne, rythme que, dans la lucidité aiguisée de la création, le peintre avait su capter et projeter sur la toile sous forme d'esquisse tracée au moyen de ces blancs de plomb et de ces bruns qui surnageaient maintenant sous l'exposition radiographique, reconstituant pour nous la vision initiale de l'œuvre et la grâce de son départ, quelque chose comme "le vers donné" de la poésie verbale, et que l'élaboration picturale avait peu à peu recouvert (Lilar, Journal 177-178; nous soulignons.)

Ce « quelque chose », invisible à l'œil nu, mais perceptible à l'aide des rayons X, d'après l'auteur, n'est rien d'autre que le rythme interne de l'œuvre d'art dont la complexité et la structure sont dues non à un simple caprice de l'auteur (en l'occurrence, le désir de faire une bonne copie), mais à une nécessité créative. Pour citer Rilke, une œuvre d'art est bonne seulement « quand elle est issue de la nécessité » (Rilke 38). Le vrai artiste, qu'il soit artiste majeur ou mineur, crée de la nécessité, de cet appel intérieur qu'est ce besoin viscéral de s'exprimer, de transmettre ou, pour parler comme Merleau-Ponty, de donner à l'invisible une forme visible qui communique le sens ${ }^{47}$. Contrairement au processus artistique d'un copiste, le vrai processus artistique demande de la part du créateur tout ensemble questionnement, tentatives de résolution et sa participation au monde extérieur et à l'être de l'œuvre en train de naître.

Le deuxième exemple concerne le célèbre Polyptyque de l'Agneau mystique (1432) des frères Hubert et Jean Van Eyck, conservé dans la cathédrale Saint-Bavon à Gand, où Suzanne Lilar a été baptisée. Lilar consacre au Polyptyque trois passages dans le Journal de l'analogiste (tous les trois se trouvent dans différents endroits) qui font écho aux souvenirs d'enfance liés à cette œuvre dans son autobiographie Une enfance gantoise. Dans ces souvenirs, l'écrivaine évoque avec tendresse ses

47 Kandinsky présente des semblables réflexions sur le processus créateur mettant en relief la notion de la « nécessité intérieure », condition d'un art véritable, selon lui: « [...] [L]'artiste a non seulement le droit, mais le devoir de manier les formes ainsi que cela est NÉCESSAIRE à ses buts. Et ni l'anatomie, ni les autres sciences du même ordre, ni le renversement par principe de ces sciences ne sont nécessaires, mais ce qui est nécessaire, c'est une liberté totalement illimitée de l'artiste dans le choix de ses moyens. » (Kandinsky 198-199) « Cette liberté illimitée doit être fondée sur la nécessité intérieure (que l'on nomme honnêteté). Et ce principe n'est pas seulement le principe de l'art, mais également celui de la vie. » (Kandinsky 199) 
premières rencontres avec la peinture de Van Eyck. Sur le plan iconique, le Polyptyque est présenté dans le Journal par un détail. Le visage de Dieu le Père figure sur deux images photographiques : l'une des illustrations est une reproduction de l'image radiographique, l'autre est le même détail en lumière directe (fig. 2).

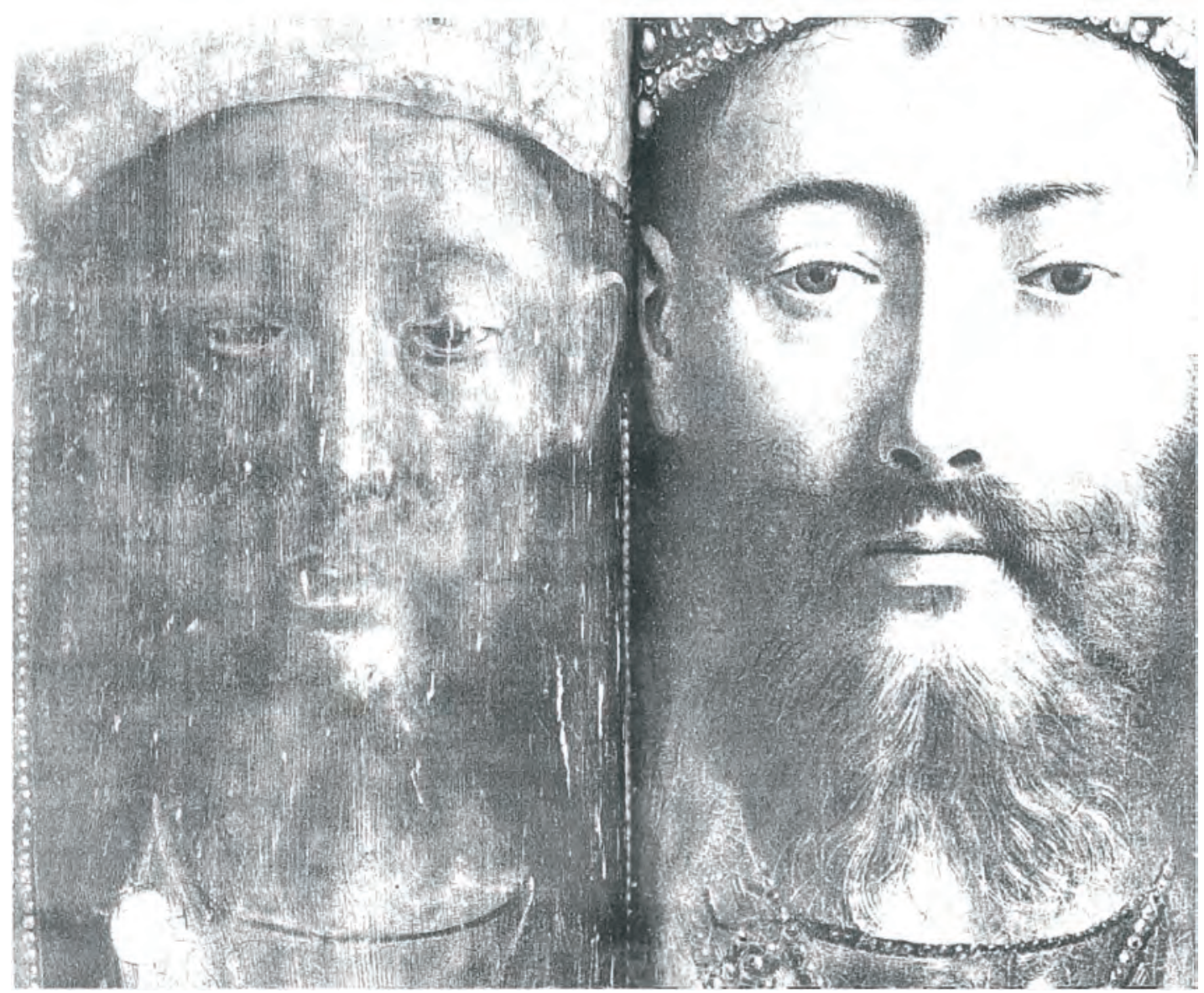

Fig. 2. Les frères Hubert et Jean Van Eyck, le visage de Dieu le Père, détail du Polyptyque de l'Agneau mystique (vers 1432). Gand: la cathédrale Saint-Bavon. Huile sur bois de chêne. L'image radiographique et l'image en lumière directe. Ces photos figurent dans les deux éditions du Journal de l'analogiste de Suzanne Lilar. 
L'auteur relate comment elle a pu apercevoir, pendant sa visite du laboratoire du Musée de B. (sans doute le Musée de Bruxelles), la correspondance entre le visible et l'invisible. Au musée, elle a pu revoir les documents de la restauration du Polyptyque, y compris ses images radiographiques :

[P]lus je comparais ces images radiographiques à la peinture, telle qu'elle nous était parvenue après cinq siècles de vieillissement et de métamorphoses, plus je me persuadais qu'elles ne se bornaient pas à nous renseigner sur l'état original de l'œuvre ni même à nous en fournir une sorte de sublime modulation, mais qu'elles nous introduisaient au cœur de son mystère. (Lilar, Journal 175-176)

Nulle part, me semblait-il, je n'avais d'aussi près appréhendé le sacré que dans ce masque divin, que le passage de la lumière blanche aux rayons $\mathrm{X}$ avait étrangement dépouillé de toute particularité d'époque ou d'origine. J'en concevais une assurance singulière, car si l'univers de Van Eyck existait si supérieurement dans l'invisible, s'il s'insérait dans les rythmes naturels d'une façon aussi irrécusable, c'est qu'il n'avait pas été arbitrairement façonné par le peintre, mais que préalable à sa recherche, il avait été par lui découvert. (Lilar, Journal 176)

Il s'agit ainsi de l'appréhension du sacré à travers l'apparition du « masque divin "sur l'image radiographique qui a rendu visible l'invisible. ${ }^{48}$ Cette expérience de l'auteur se perçoit également comme une initiation "pratique" à la poésie qui s'insère "dans les rythmes naturels » du monde. Or il n'est pas toujours nécessaire de recourir aux « moyens scientifiques pour surprendre la parenté des ouvrages de la poésie avec les rythmes naturels » (Lilar, Journal 178).

48 Il n'est pas inutile de rappeler ici la célèbre formule de Paul Klee : « L'art ne reproduit pas le visible, il rend visible. » (Klee 34$)$ 


\section{Un détail du retable Heller}

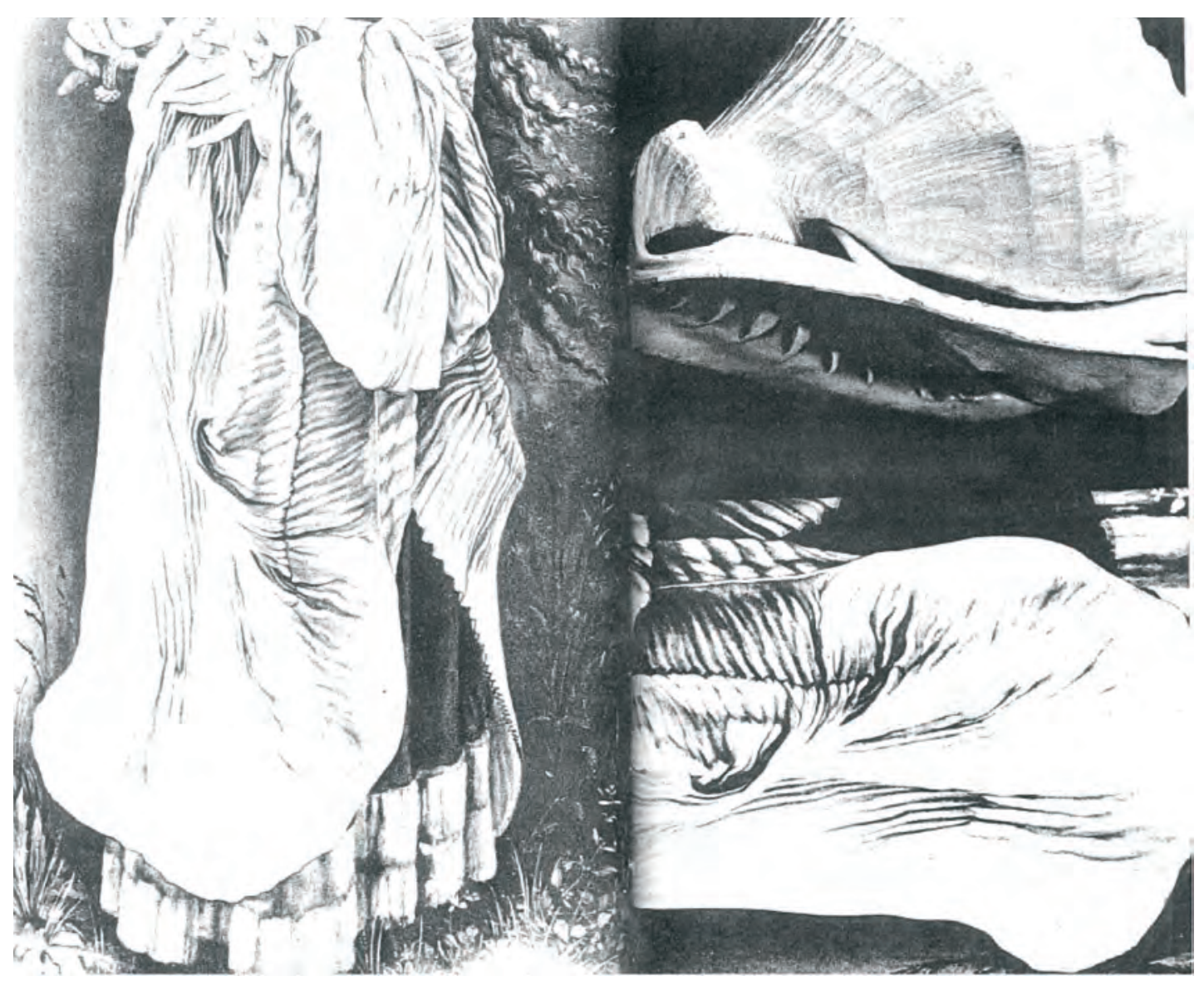

Fig. 3. Confrontation de détails agrandis de la robe de sainte Lucie, détail de la peinture en grisaille sur un volet du retable Heller, avec un coquillage: le "tissu rythmique" (Lilar, Journal 192) de l'objet artistique se trouve en harmonie avec la "pulsation organique" (Lilar, Journal 193) de la matière. Ce montage photographique est présent dans les deux éditions du Journal de l'analogiste de Suzanne Lilar. 
Révélatrices à cet égard sont les observations que fait l'écrivaine belge en confrontant un détail du retable Heller, œuvre de Dürer et de Grünewald, un coquillage qui lui servait de presse-papier. Lilar aperçoit une parenté frappante entre le «plissement » (Lilar, Journal 180) de la robe de sainte Lucie (détail de la peinture en grisaille sur un volet du retable) et la forme du coquillage (fig. 3 et 4). Étonnée de cette ressemblance, Lilar voulait savoir " s'il s'agissait d'un hasard subi par le peintre ou, au contraire, d'une réalité génialement pressentie » (Lilar, Journal 179). À cette fin, elle commande des photographies du coquillage et de " cette robe bizarrement tournée et contournée, plissée et déplissée » (Lilar, Journal 180). Quelques-unes de ces photographies sont insérées dans le Journal de l'analogiste. La confrontation des détails agrandis a donné un résultat dépassant toutes les attentes :

Fig. 4. Confrontation de détails agrandis de la robe de sainte Lucie avec un coquillage. Ces photos figurent dans les deux éditions du Journal de l'analogiste.

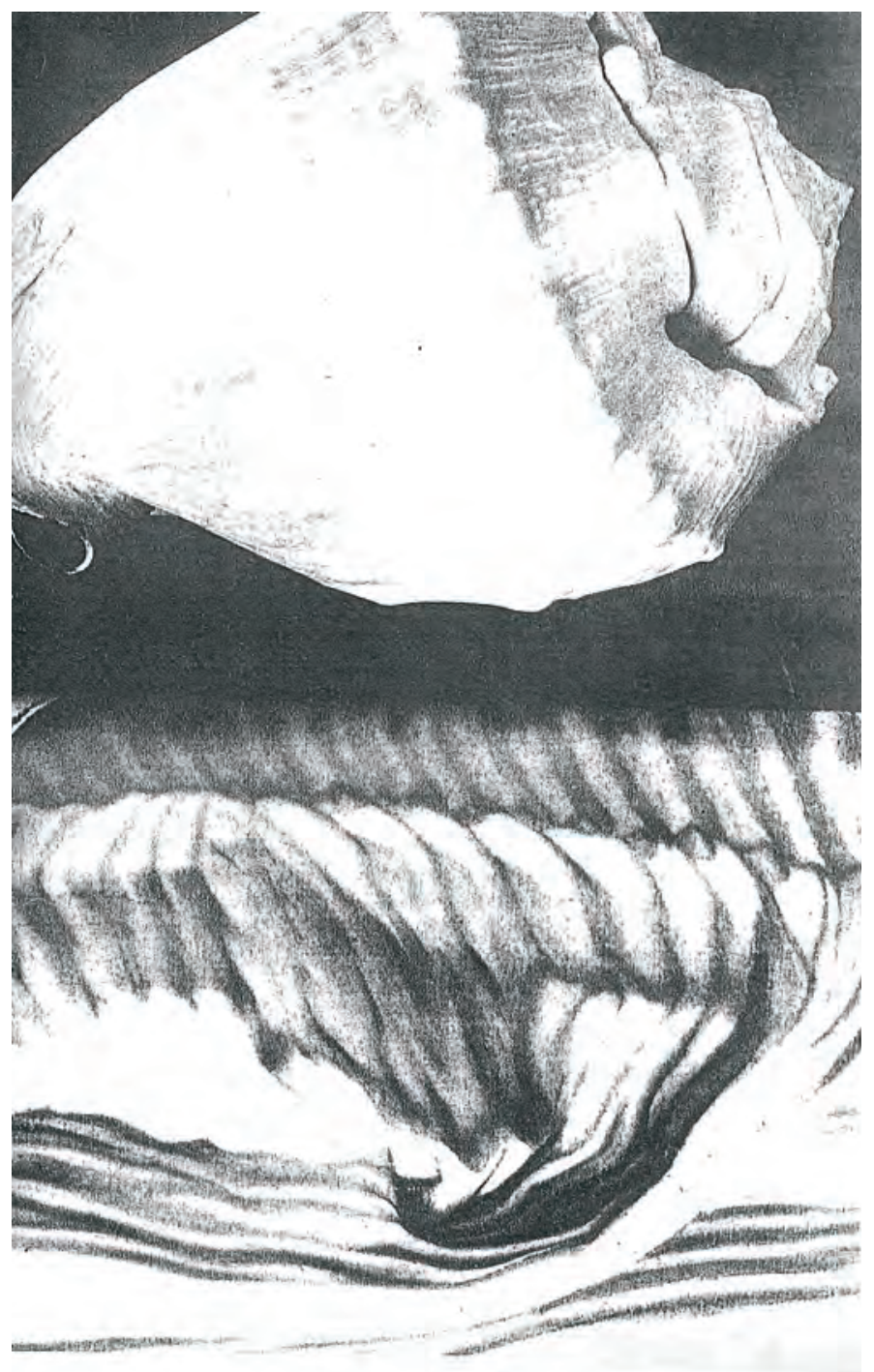


Non seulement la robe se développait et s'infléchissait en forme de valve, [...] mais encore son tuyauté imitait le plissement du test, cependant que les cassures de l'étoffe ou ses coutures reproduisaient avec une précision stupéfiante la suture des tours avec leurs cordons de nodosités nommées tubercules. [...] Mais plus mystifiante encore dans son analogie tout interne m'apparaissait la manière que cette robe avait de tourner autour d'un axe invisible et de se déployer en hélice. En vérité, plus je comparais cet objet et cette forme [...], plus je me sentais encouragée à penser qu'à partir d'un certain degré d'élaboration, de perfection artisanale, le poète opère sa jonction avec les rythmes naturels. Non qu'au niveau de ses émotions, il n'en détienne déjà le module [...] mais pour se muer en poésie, ce rythme personnel demandait à travers la forme à fusionner avec d'autres rythmes, à affirmer ses parentés, à se reconnaître pour universel. (Lilar, Journal 180-181)

Ainsi, en faisant fonctionner l'analogie dans le domaine de la création artistique et dans la vie quotidienne, Lilar découvre des correspondances entre les rythmes et les formes de la nature et les rythmes et les formes de l'art. Elle s'émerveille devant l'harmonie du monde où toutes les choses se tiennent et communiquent dans un échange mutuel.

\section{Le Polyptyque de l'Agneau mystique : rencontres et découvertes}

Le Polyptyque de l'Agneau mystique fait partie de ce que Suzanne Lilar appelle « ma mythologie personnelle » (Lilar, Journal 96). Ce retable se situe au cœur de la formation sensuelle et spirituelle de l'écrivaine et participe effectivement à l'élaboration de sa théorie des correspondances. Devant ce chef-d'œuvre, elle passe des heures ; littéralement, elle « entr[e] dans la peinture » (Lilar, Journal 211). Ayant médité sur la vocation du Polyptyque dans sa vie, Lilar met des éléments du tableau dans leur rapport particulier avec sa personnalité. Aux différents moments de sa vie, la peinture de Van Eyck a révélé à Lilar des choses différentes. L'espace pictural est sans doute pluriel : il y a toujours «l'opposition de la patence et de la latence» (Escoubas 35). Ainsi, ce qu'on voit en regardant le tableau n'est jamais effectivement vu, mais « quasiment vu » (id., 21-22). Pour Suzanne écolière, le Polyptyque n'était que « cette peinture dont on regarde les fleurs à la loupe et dont les personnages ont un air de famille » (Lilar, Journal 204). En effet, le spectateur peut identifier dans le paysage paradisiaque représenté par Van Eyck quarante-deux espèces de plantes. Tout, dans cette prairie, comme l'écrit Lilar « resplendi[t] de la fraîcheur et de l'évidence de l'archétype » (Lilar, Enfance gantoise 134). En outre, là, dans l'espace pictural du Polyptyque, parmi les personnages de la composition, la petite Suzanne retrouve le visage de sa mère. Elle le retrouve et ne le projette pas : 
Le jeu des ressemblances, écrit Lilar, est plein de magie. Pour ma part j'ai toujours cru qu'elles impliquent un lien secret et il ne me paraît pas exclu qu'elles inclinent à la poésie. Souvent déjà j'avais été frappée par un air de famille entre le visage de ma mère et celui des personnages de van Eyck. Dans la foule du panneau central du Polyptyque, je la retrouvais parmi les Anges mais aussi les Philosophes, les Ermites et sur le panneau des Juges intègres jusque dans le portrait d'Hubert lui-même. (Lilar, Journal 119)

Comme dans le cas de la sainte Lucie de Grünewald, il s'agit de la fusion des rythmes personnels de l'artiste avec ceux du spectateur : le multiple tend à l'Un et, inversement. Les rythmes individuels épousent les rythmes universels sous « l'afflux nourricier d'une circulation poétique » (Lilar, Journal 192) naissant du dialogue entre le tableau et le sujet regardant. Lilar souligne que l'un des privilèges des grandes œuvres est « d'offrir un système d'analogies si riche, si foisonnant qu'il ne cess[e] de se renouveler » (Lilar Journal 143) :

Mais après les visages, c'était aujourd'hui la composition tout entière qui venait réveiller en moi les plus doux souvenirs, car la prairie de l'Agneau, avec son moelleux et dru gazon des Flandres, était cet herbage proche de la Lys où, me trouvant à l'âge de onze ans avec mes compagnes de classe, j'avais dû m'écarter du groupe afin de dissimuler mes larmes. Et la présence sur la toile de l'agneau au cœur percé n'offrait pas plus de tranquille évidence que n'en avait eu ce jour-là pour moi la présence de Dieu. Mais il m'avait fallu bien des années pour la reconnaître, la prairie mystique, car si je savais, lorsque la classe entière se déplaçait pour aller contempler le chef-d'œuvre, identifier au travers de la loupe la fleur de fraisier, l'ancolie et la violette, [...] je n'étais pas alors en mesure de démêler toutes les correspondances secrètement tissées entre cette peinture et moi bien avant ma naissance. (Lilar, Journal 119-120; nous soulignons.)

Les phrases de ce passage (mises en italique) où le verbe reconnaître (on songe à la co-naissance de Claudel) est le mot-clé nous plongent directement au cœur du monde des Idées de Platon. Or, pour que ce verbe se remplisse de sens, il faut le temps : pour certains, cela prend des années, pour les autres, des mois ou des jours. C'est en ce sens que l'écrit Rilke, dans une de ses lettres au jeune poète Franz Kappus :

[J]'aimerais vous prier [...] d'avoir patience envers tout ce qu'il y a de non résolu dans votre cœur et d'essayer d'aimer les questions elles-mêmes comme des chambres verrouillées, comme des livres écrits dans une langue très étrangère. Ne partez pas maintenant à la recherche 
de réponses qui ne peuvent pas vous être données parce que vous ne pourriez pas les vivre. Et ce dont il s'agit, c'est de tout vivre. Vivez maintenant les questions. (Rilke 55)

Ainsi, se trouvant devant le Polyptyque sous des circonstances différentes, Lilar « vivait ses questions » avec une ardeur digne des grands mystiques flamands. Or, il a fallu bien des années pour que la poésie l'affranchisse « des conditions habituelles de la perception » et qu'elle re-connaisse la prairie mystique, sa prairie gantoise. Mais comment est-il possible de re-co-nnaître quelque chose qu'on avait déjà vu maintes fois, c'est-à-dire quelque chose que l'on connaît ? Bien plus, comment est-ce que l'auteur belge a pu reconnaître et « démêler » les liens secrets qui l'attachent à la peinture de Van Eyck « bien avant [sa] naissance ? $»^{49}$ Enfin, comment a-t-elle pu retrouver le visage de sa mère à la fois chez les Anges, chez les Philosophes et chez les Ermites ? Mais c'est « le paradoxe de la poésie, aurait pu répondre Lilar, que, nous délivrant du particulier, elle ne [...] s'exerc[e] que sur lui » (Lilar, Journal 166).

D'après Lilar, appréhender l'œuvre d'art n'est possible qu' " à travers l'expérience du poète » (Lilar, Journal 218). C'est bien en poète qu'elle contemple un tableau, une statue, une fleur ou écoute de la musique. Il s'agit en effet de la « contemplation poétique » (Lilar, Journal 158), de «l'appropriation » des formes poétiques, qu'elles soient artistiques ou naturelles. Cette " appropriation » passe par la transfiguration des formes représentées et s'accroche aux représentations mentales, souvenirs, expériences concrètes. L'œuvre d'art demande une adhésion de la part du spectateur. L'œuvre touche individuellement et différemment, car « [c]hacun de nous, comme l'écrit Lilar, [a] ses repères ». (Lilar, Journal 93) C'est lorsque le spectateur éprouve quelque chose devant l'objet d'art que la poésie « intervient » (Lilar, Journal 96). Elle est le fruit de rapports dialogiques et ne peut être acquise une fois pour toutes. Le dialogue doit être constamment renouvelé. C'est pour cela que Lilar constate que « le pouvoir poétique d'un poème, d'une musique, d'un tableau n'[est] pas inépuisable » (Lilar, Journal 142), mais la poésie « s'évente » (142).

Contrairement à la beauté qui « est dans les choses » (Lilar, Journal 98), la poésie «n’est pas un prêt-à-consommer » (Gracq), « c’est nous qui y projetons la poésie » (Lilar, Journal 98) :

49 On retrouve les mêmes idées également chez Henri Maldiney, par exemple, dans « Le faux dilemme de la peinture » : abstraction ou réalité": « Tout tableau est un événement; et il l'est dans la mesure où il nous offre une surprise. Ici, surprise de voir surgir d'un Rythme unique, imprévu, créé dans l'Instant même, une impression familière reconnue $[. .$.$] qui semble exister depuis toujours parce que l'Instant rythmique où il vient à l'existence$ est un présent éternel”. (Maldiney, « Le faux dilemme de la peinture » 6). 
$[C]$ hacun de nous est réduit à distiller sa propre poésie. Image ou rythme attendaient eux aussi le miracle d'une rencontre. Ils aspiraient à être reconvertis, à fusionner avec de nouvelles formes sensibles que nous étions appelés à leur fournir. Faute de cette contribution, leur pouvoir réducteur ne trouvait pas à s'exercer et leur poésie demeurait pour nous lettre morte. (Lilar, Journal 121)

En réalité, l'œuvre d'art peut être belle et pourtant non poétique. L'art « détourné de la poésie » (Lilar, Journal 114) ne fait que nous contenter, il ne déclenche aucun questionnement ni aiguise le regard, il ne remet non plus en question notre rapport au monde. Tandis que la poésie « commen[ce] par nous arracher au bien-être, par nous mettre en état d'alerte », parce qu'elle n'est " qu'accès, ouverture » (114). Cette « ouverture » permet à Lilar de vivre certains moments privilégiés dans lesquels elle perçoit un autre monde au-delà du terrestre. Le dialogue que l'auteur mène avec les œuvres d'art pendant toute sa vie lui sert comme un " moyen supplémentaire de saisir la vie » (Rilke 42) dans toute sa richesse et sa complexité évolutive. Comme le note Paul Klee, des « réalités de l'art " " élargissent les limites de la vie telle qu'elle apparaît d'ordinaire » justement parce que ces réalités « ne reproduisent pas le visible avec plus ou moins de tempérament, mais rendent visible une vision secrète » (Klee 31). Au fond, l'expérience artistique et poétique ne nous détournent aucunement de la vie de tous les jours, mais « éveillent à la réalité » (Maldiney, L'Art 95). Ce n'est pas un hasard si, au seuil du livre, l'auteur belge met en exergue les formules suivantes de Novalis : « La poésie est le réel absolu. [...] Plus une chose est poétique, plus elle est réelle ».

\section{Conclusion}

En proposant une approche poétique au monde artistique, le Journal de l'analogiste nous éloigne de la vision banalement utilitaire du philistin, dont le regard lourd « pèse » sur le monde contemporain " comme un couvercle » (Baudelaire, LXII. « Spleen », vers 1). L'ouvrage met en évidence le rôle puissant de médiation de l'image qui se présente non seulement comme un appui du savoir, mais aussi comme sa composante motrice. C'est moyennant l'art, moyennant la peinture notamment, que l'écrivaine belge oriente le lecteur vers le questionnement sur l'Être, fondé sur l'entrelacement poétique du visible et de l'invisible. Le texte révèle des aspects sous-jacents à la performance dialogique entre l'œuvre d'art et le sujet regardant. Ce dialogue, cet échange mutuel est lié indissolublement à l'expérience poétique du spectateur qui passe par des recréations et réflexions analogiques en se ressourçant aux souvenirs personnels, expériences vécues, images entrevues et retenues. En outre, il ne cesse jamais, il débouche sur l'ouverture et dévoile des correspondances entre le particulier et le général, entre l'unique et le multiple, 
entre les rythmes et formes naturels et les rythmes et formes artistiques. Les positions que prend ici Lilar vis-à-vis de l'objet artistique rejoignent celles d'un Maldiney ou d'un Merleau-Ponty, pour lesquels l'œuvre d'art est vouée à une renaissance perpétuelle et ne peut constituer une « téléologie du sens ».

Pour conceptualiser son expérience poétique, l'auteur fait recours à la reproduction radiographique et photographique des détails du Polyptyque de Van Eyck et ceux du retable Heller de Grünewald. Cette démarche fait penser inévitablement à la capacité qu'a la photographie de morceler l'objet d'art, de le fragmenter, et ainsi de le transformer. On ne peut ne pas prononcer, dans ce contexte, le nom de Walter Benjamin dont les textes insistent à maintes reprises sur la dimension destructrice de l'image photographique qui fait «périr l'aura » (Benjamin 273-276) de l'œuvre d'art, car il la prive de ses valeurs d'authenticité et de son intégrité, signe du déclin de l'art dans le monde moderne. Cependant, dans cet ouvrage, ces « morcèlements » et « agrandissements » photographiques remplissent plutôt les fonctions épistémologiques, car ils révèlent ce que l'œil ne voit pas. Par ailleurs, cette mise en fragments est en harmonie complète avec le texte même du Journal, fragmentaire par définition.

Au demeurant, il importe de dire que dans le Journal de l'analogiste, l'image et le texte à la fois se confrontent et collaborent. En lisant et regardant, le lecteur perçoit la théorie en train de s'élaborer (métapicturalité [terme de Liliane Louvel]) : l'image commente le texte et réciproquement. Pour paraphraser Henry Maldiney, c'est le cas où l'acte par lequel une forme se forme est aussi celui par lequel elle nous informe et communique un sens.

Ainsi, en renouvelant l'héritage culturel de la civilisation dite occidentale (qu'il s'agisse de l'ontologie de Platon ou de la poésie du béguinage flamand des XIII ${ }^{\mathrm{e}}-\mathrm{XV}^{\mathrm{e}}$ siècles [Hadewijch, Marguerite d'Ypres, Ida de Louvain] ou encore des correspondances de Baudelaire), le Journal de l'analogiste de Suzanne Lilar rapproche la poésie, l'art, la philosophie et l'existence : la poésie démasque des situations fausses auxquelles nous faisons face au quotidien et donne l'accès à l'essence des choses et des êtres. Cet ouvrage, illustré par la photographie, s'impose sur la scène bien particulière des lettres belges par son originalité et sa profondeur philosophique, les investissant de nouvelles dimensions. Il n'est donc pas surprenant que dans ses Balises pour l'histoire des lettres belges Marc Quaghebeur, grand connaisseur de la littérature belge, classe à part l'œuvre de Lilar, la mettant sous la rubrique « la vague néo-classique ». Éminemment « irrégulière », elle se démarque également par sa puissance poétique qui permet de conjuguer harmonieusement l'esprit métaphysique flamand et la rigueur des moralistes et écrivains français du XVII ${ }^{\mathrm{e}}$ siècle : toute poésie, selon l'écrivaine belge, nous «mèn[e] en un lieu où les contraires cessent d'être perçus contradictoirement » (Lilar, Journal 137), à cet extrême point terrestre de notre Être qui touche déjà le Ciel et qui s'approche de Dieu. 


\section{Ouvrages Cités}

BAUDELAIRE, Charles. LXII. « Spleen », Les Fleurs du mal. 1857. Anjou (Québec) : Les éditions CEC, 2015, coll. Grands textes.

BENJAMIN, Walter. « Das Kunstwerk im Zeitalter seiner technischen Reproduzierbarkeit ». 1939. Français : « L'œuvre d'art à l'ère de sa reproductibilité technique ». Traduit de l'allemand par Maurice de Gandillac, Pierre Rusch et Rainer Rochlitz. Euvres III. Paris : Gallimard, coll. folio essais nº 374, 2000. 269-316.

ESCOUBAS, Éliane. L'Espace pictural, Fougères/La Versanne : Encre marine, 1995.

GRACQ, Julien. "Préface”. In Journal de l'analogiste. Paris : Bernard Grasset, 1979. 11-17.

KANDINSKY, Wassily. О духовном в искусстве. 1910. Français : Du spirituel dans l'art, et dans la peinture en particulier. Traduit du russe par Bernadette du Crest. Paris : Éditions Denoël, coll. Folio essais no 72, 1989.

KLEE, Paul. Pädagogisches Skizzenbuch. 1925. Français : Théorie de l'art moderne. Traduit de l'allemand par Pierre-Henri Gonthier. Paris : Denoël, coll. Folio essais nº 322, 1985.

LILAR, Suzanne. Journal de l'analogiste. Paris : Julliard, 1954.

. Journal de l'analogiste, préface de Julien Gracq, introduction de Jean Tordeur. Paris : Bernard Grasset, 1979. . Une enfance gantoise. Paris : Bernard Grasset, 1976.

. Inédits de Suzanne Lilar : écrits à caractère autobiographiques : fragments divers : Lilar sur elle-même et sur son œuvre (08492/0024), Archives et Musée de la Littérature (AML) de la Bibliothèque Royale de Belgique, Bruxelles.

LOUVEL, Liliane. L'Eil du texte, Toulouse : Presses Universitaires du Mirail, 1998.

MALDINEY, Henri. « Le faux dilemme de la peinture : abstraction ou réalité ». Regard. Parole. Espace. Lausanne : Éditions de l'Âge d'Homme, 1973. 1-20. . «L'esthétique des rythmes ». Regard. Parole. Espace. Lausanne : Éditions de l'Âge d'Homme, 1973. 147-172. . L'Art, l'éclair de l'être. Paris : Éditions Comp'Act, coll. Scalène, 1993.

MARIN, Louis. De la représentation. Paris : Gallimard / Le Seuil, 1994.

MERLEAU-PONTY, Maurice. Le Visible et l'Invisible, Euvres, édition établie et préfacée par Claude Lefort. Paris : Gallimard, coll. Quarto, 2010. 1629-1787.

RILKE, Rainer Maria. Briefe an einen jungen Dichter. Français : Lettres à un jeune poète, Traduit de l'allemand par Claude Porcell. Paris : Flammarion, 1994.

TORDEUR, Jean. «Introduction ». In Journal de l'analogiste. Paris : Bernard Grasset, 1979. 21-68.

WUNENBURGER, Jean-Jacques. Philosophie des images. Paris : Presses Universitaires de France, coll. Thémis, 1997. 


\section{QEUVRES CITÉES}

GOYA, Les Vieilles (1820), conservée au Palais des Beaux-Arts de Lille. Huile sur toile.

GRUNEWALD et DÜRER, Retable Heller (vers 1509-1510) Musée Städel, Francfort-sur-le-Main et Kunsthalle, Karlsruhe.

REMBRANDT, Portrait de Hendrickje Stoffels (vers 1654-1656), conservé à Londres, National Gallery. Huile sur toile.

Les frères Hubert et Jean VAN EYCK, Polyptyque de l'Agneau mystique (vers 1432). Gand : la cathédrale SaintBavon. Huile sur bois de chêne. 